\title{
Prevalence of Staphylococcus aureus among clinical isolates and their responses to selected antibiotics at Centre Hospitalier Universitaire de Kigali (CHUK)
}

\begin{abstract}
Staphylococcus aureus is one of the major causes of bacteremia associated with higher morbidity and mortality, compared to the bacteremia caused by other pathogens. The burden of $S$. aureus bacteremia, particularly penicillin-resistant $S$. aureus, in terms, of cost and resource use is high. $S$. aureus, a mainly acquired hospital infection is responsible for many suppurative lesions and has demonstrated the ability of developing resistance to many antimicrobial agents leading to life threatening infections and long hospital stay. A cross-sectional study was carried out at CHUK to determine the prevalence and antibiotic susceptibility profiles of $S$. aureus isolates from blood and pus samples. After gram staining, cultural techniques on Mannitol Salt Agar (MSA) and blood agar were performed. Staphylococcus aureus was isolated based on the colonial characteristics and confirmed by Catalase and Coagulase tests. The antibiotic susceptibility test was done on Mueller Hinton agar (MHA) by disc diffusion method.
\end{abstract}

A total of 300 specimens were collected over a period of three months. Out of 300 specimens, $30(10 \%)$ and $270(90 \%)$ proved to be positive and negative respectively for $S$. aureus. Among the 30 positive for $S$. aureus, 11(36.7\%) and 19(63.3\%) were from blood and pus respectively. The sensitivity pattern for the 6 antibiotics tested was: Vancomycin (8.5\%), Penicillin (4.9\%), Erythromycin (18.3\%), Oxacillin (10.97\%), Clindamicyn $(32.9 \%)$ and Tetracycline $(24.4 \%)$. The resistance at CHUK was high for the penicillin 24 $(27 \%)$ and vancomycin $21(24 \%)$

There is a higher risk of acquiring multidrug resistant Staphylococcus aureus infection in inpatients attending CHUK, thus strategies need to be adopted in order to stop an increasing resistance of $S$. aureus to different antibiotics due to high cost and time in developing new antibiotics.

Keywords: prevalence, Staphylococcus aureus, antibiotic susceptibility test, cultural and Biochemical tests
Volume 5 Issue 4 - 2017

\author{
Gahamanyi Noel,' Bitariho Benigne, ${ }^{2}$ Muhire \\ Vincent ${ }^{3}$ \\ 'Lecturer in Biomedical Laboratory Sciences, Catholic \\ University of Rwanda, Rwanda \\ ${ }^{2}$ Student in Biomedical Laboratory Sciences, Catholic University \\ of Rwanda, Rwanda \\ ${ }^{3}$ Laboratory Technician, CHUK
}

Correspondence: Gahamanyi N, Department of Biomedical Laboratory Sciences, Faculty of Science and Technology, Catholic University of Rwanda, PO Box 49 Butare/Huye-Rwanda, Rwanda, Tel 250788829833, Email gahanoel 1984@gmail.com

Received: July 27, 2017| Published: August 09, 2017
Abbreviations: MSA, mannitol salt agar; MHA, mueller hinton agar; MRSA, methicillin-resistant S. aureus; AST, antimicrobial susceptibility testing

\section{Introduction}

$S$. aureus is an aerobic gram positive coccus and ubiquitous commensal that periodically lives on the skin and anterior nares of about one third of the healthy human population without causing illness. $^{1-3} S$. aureus is a leading cause of diseases like skin and soft tissue infections, pneumonia, bloodstream infections, osteomyelitis and endocarditis, as well as toxin-mediated syndromes like toxic shock and food poisoning in many developed countries. ${ }^{4,5}$ S. aureus has been the major cause of morbidity and mortality, and is among the ten main causes of death worldwide and the basic cause of death in $1 \%$ of cases. ${ }^{6}$

Antimicrobial agents have been used extensively to combat $S$. aureus infections but the increasing level of resistance of $S$. aureus to many antibiotics is complicating the treatment of serious infections caused by this pathogen. ${ }^{7}$ Antibiotics exert a selective pressure which plays a central role on the acquisition, selection, persistence and transmission of resistant pathogens.
S. aureus represents a prototype for drug resistance, especially to $\beta$-lactam antibiotics. Although this bug has been naturally susceptible to almost every antibiotic developed so far, it frequently gains resistance by gene mutations and horizontal gene transfer, that protect the bug under antibiotic selection pressure, and has been implicated in episodes of epidemic and pandemic proportions. ${ }^{1,8}$

Two years after the massive use of penicillin to treat bacterial infections in the 1940 s, strains of $S$. aureus able to produce penicillinase were selected and in 1960, almost 100\% of strains were already resistant to penicillin. The discovery of semisynthetic penicillins (Methicillin and oxacillin) resistant to $\beta$-lactamase hydrolysis was achieved and the two drugs were used to treat Staphylococcal infections in the early 1960s. ${ }^{9,10}$ Unfortunately, after one year, resistance to methicillin was noticed in Europe and North America, and then worldwide. ${ }^{11}$

Adult patients without serious complications can be given trimethoprim-sulfamethoxazole (TMP-SMX), minocycline, doxycycline, or clindamycin, Ampicillin, Chloramphenicol, Ciprofloxacin, Erythromycin, Gentamycin, Methicillin, Tetracycline and Co-trimoxazole. ${ }^{12}$

The rise of drug-resistant and virulent strains of $S$. aureus, particularly methicillin-resistant $S$. aureus (MRSA) is a serious 
problem in the treatment and control of Staphylococcal infections. ${ }^{13}$ Efforts to eradicate carriage of $S$. aureus are one of the strategies adopted by control programs against the spread of MRSA. ${ }^{14}$ The knowledge of antimicrobial susceptibility profile of $S$. aureus in a particular area is important as this can contribute to rational choice and use of antimicrobial agents. ${ }^{15}$

\section{Material and methods}

A total of 300 non-duplicate Specimens were collected from CHUK between April and July 2014. The clinical specimens used were from blood and pus samples collected using sterile cotton swabs impregnated with sterile normal saline solution.

All isolates were characterized and identified by Gram staining, Catalase and coagulase tests. Cultural characteristics including golden yellow colonies of $S$. aureus on Mannitol Salt Agar (MSA) were used in $S$. aureus identification. After inoculation, plates were incubated at $370 \mathrm{C}$ for 24 hours and then read. ${ }^{16,17}$ At $100 \mathrm{X}$, S. aureus appears as purple gram-positive cocci in clusters. . $^{16,18,19}$

Slide coagulase test was performed by emulsifying few pure colonies of Staphylococci from Blood agar with undiluted plasma. Agglutination within seconds indicated a positive result. Staphylococcal isolates negative by slide coagulase test were again checked for tube coagulase test by diluting the plasma with normal saline (1:6) and the tubes were examined after four hours. If negative, the tubes were further incubated overnight at room temperature. Clotting of the plasma represents positive result. ${ }^{20}$

The antimicrobial susceptibility testing (AST) was done using the Kirby-Bauer disc agar diffusion method on MHA with commercially available antibiotic sensitivity discs (AbtekBiologicals, Ltd, UK). The procedure of the AST was done as described by Ekundayo et al. ${ }^{21}$ After incubation, the plates were examined and the zone of inhibition was measured. The results were interpreted as per CLSI standards (CLSI, 2011). The data were presented in form of tables and complimented with columns and analyzed using Microsoft Office to get the prevalence and AST of $S$. aureus isolates during the study period.

\section{Results}

Results were presented based on age. The following table summarizes results based on presence or absence of $S$. aureus and the age of the patient. Table 1 shows that the interval with both higher positive and negative results is ${ }^{20-29}$ with $43.3 \%$ and $32.5 \%$ respectively. The Table 1 also shows that 30 patients had $S$. aureus infection and 270 were free from it. In addition, results were presented based on gender of patients as follow (Table 2).

Table I Prevalence of S. aureus based on age

\begin{tabular}{|c|c|c|c|c|c|c|}
\hline \multirow{2}{*}{$\begin{array}{c}\text { Results } \\
\text { Ages (yrs) }\end{array}$} & \multicolumn{2}{|l|}{ Positive } & \multicolumn{2}{|l|}{ Negative } & \multirow{2}{*}{-Total } & \multirow{2}{*}{$\%$} \\
\hline & Number & $\%$ & Number & $\%$ & & \\
\hline$<10$ & 0 & 0 & 4 & 1.5 & 4 & 1.5 \\
\hline $11-20$ & 11 & 36.7 & 80 & 30 & 91 & 30.3 \\
\hline $21-30$ & 13 & 43.3 & 88 & 32.5 & 101 & 33.6 \\
\hline $31-40$ & 1 & 3.3 & 75 & 27.7 & 76 & 25.3 \\
\hline $41-50$ & 4 & 13.3 & 23 & 8.5 & 27 & 9 \\
\hline$<50$ & 1 & 3.3 & 0 & 0 & 1 & 0.3 \\
\hline Total & 30 & 100 & 270 & 100 & 300 & 100 \\
\hline
\end{tabular}

Table 2 Prevalence of S. aureus based on gender

\begin{tabular}{|c|c|c|c|c|c|c|}
\hline \multirow{2}{*}{ Results sex } & \multicolumn{2}{|l|}{ Positive } & \multicolumn{2}{|l|}{ Negative } & \multirow{2}{*}{-Total } & \multirow{2}{*}{$\%$} \\
\hline & Number & $\%$ & Number & $\%$ & & \\
\hline Female & II & 36.7 & III & 41.1 & 122 & 40.7 \\
\hline Male & 19 & 63.3 & 159 & 58.9 & 178 & 59.3 \\
\hline Total & 30 & 100 & 270 & 100 & 300 & 100 \\
\hline
\end{tabular}

Table 3 Distribution of results based on type of specimen

\begin{tabular}{lllllll}
\hline Results & Positive & \multicolumn{3}{c}{ Negative } & \multirow{2}{*}{ Total } \\
\cline { 2 - 6 } specimen & Number & $\%$ & Number & $\%$ & & 190 \\
Blood culture & II & 36.7 & 179 & 66.3 & 63 & 17 \\
Pus samples & 19 & 63.3 & 91 & 33.7 & 37 & 110 \\
Total & 30 & 100 & 270 & 100 & 100 & 300 \\
\hline
\end{tabular}

Table 4 Antibiotic susceptibility pattern of S. aureus isolates

\begin{tabular}{lllllll}
\hline Results & Sensitive & \multicolumn{3}{c}{ Intermediate } & \multicolumn{2}{c}{ Resistant } \\
\cline { 2 - 7 } antibiotics & Number & $\%$ & Number & $\%$ & Number & $\%$ \\
\hline Erythromycin & 15 & 18.3 & 0 & 0 & 14 & 16 \\
Tetracycline & 20 & 24.4 & 0 & 0 & 9 & 10 \\
Vancomycin & 7 & 8.5 & 1 & 33.3 & 21 & 24 \\
Penicillin & 4 & 4.9 & 1 & 33.3 & 24 & 27 \\
Oxacillin & 9 & 10.97 & 1 & 33.3 & 19 & 21 \\
Clindamycin & 27 & 32.9 & 0 & 0 & 2 & 2 \\
Total & 82 & 100 & 3 & 100 & 87 & 100 \\
\hline
\end{tabular}


Of the 300 patients enrolled, 178(59.3\%) and 122(40.7\%) were males and females respectively. Males and females with $S$. aureus were $19(63.3 \%)$ and $11(36.7 \%)$ respectively (Figure 1). Furthermore, results were also presented based on the type of Specimen (Table 3). Of the 300 specimens collected, 190(63.0\%) and 110(37.0\%) were collected from blood culture and pus samples respectively. The Specimens with $S$. aureus infection were 11(36.7\%) in blood and 19(63.3\%) in pus samples (Figure 2). Specimens were tested for the susceptibility to different antibiotics. The following table gives a summary of the results obtained (Table 4).

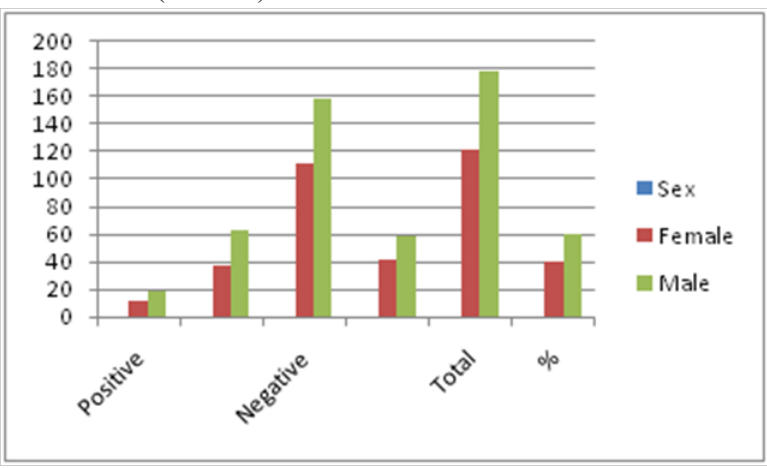

Figure I Prevalence of S. aureus based on gender.

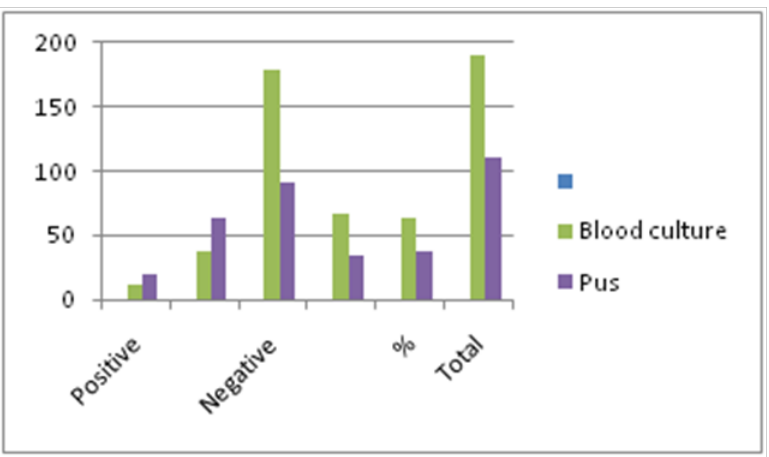

Figure 2 Distribution of results based on specimen.

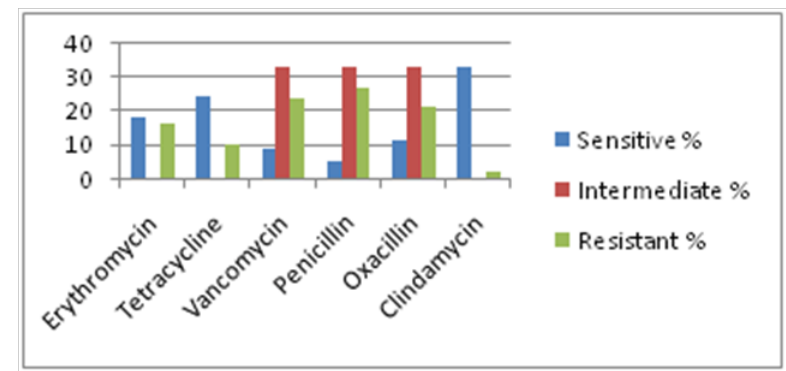

Figure 3 Antibiotic susceptibility pattern of S. aureus isolates.

The results showed that $S$. aureus isolates were sensitive to clindamycin with $27(32.9 \%)$, followed by tetracycline with $20.0(24.4 \%)$ and the erythromycin with $15(18.3 \%)$. The above table also showed that the isolates were resistant to Penicillin with $24(27 \%)$ followed by Vancomycin with 21(24\%), and then Oxacillin 19(21\%). Note that the Antibiotic Susceptibility Test was done for 29 isolates and not 30 (Figure 3).

\section{Discussion}

The study showed that the overall prevalence of $S$. aureus was $10 \%$ which is less than the results of earlier studies with a prevalence of $40.5 \%{ }^{22}$ and $24.5 \%{ }^{23}$ Although the results of this study showed a lower prevalence of $S$. aureus when compared to other studies, $S$. aureus should be considered as a pathogen of great concern.

Of the 30 isolates positive for S. aureus, 11(36.7\%) and 19(63.3\%) were from Blood and pus swabs respectively which concurs with $1(6.7 \%)$ and $10(29.4 \%){ }^{23}$ The resistance of $S$. aureus to several antimicrobial agents represents a serious concern. The organisms exhibit remarkable versatility in their behavior towards antibiotics, with some strains having become resistant to most commonly used antibiotics. $^{24}$

Antimicrobial resistance patterns of $S$. aureus infection in the present study showed that $S$. aureus isolates were sensitive to tetracycline with $20.0\left(24.4 \%\right.$ ) which is in accordance with $16(69.6 \%)^{24}$ but contrasts with $0(0 \%){ }^{25}$ Furthermore, our results showed that $15(18.3 \%)$ S. aureus isolates were sensitive to Erythromycin which concurs with $49(47.1 \%){ }^{23}$

On the other hand, our results proved that 24(27\%), 19(21\%) were resistant to Penicillin and Oxacillin respectively which is in accordance with 66(90\%), 32(43.8\%) $)^{23}$ and 462(100), 462 (100) for Penicillin and Oxacillin respectively. ${ }^{25}$ High rate of resistance to penicillin and other $\beta$-lactam antibiotics has been reported in parts of South-western Nigeria. ${ }^{26-28}$ The high level of resistance to the two antibiotics may be attributed to overuse of them as empirical treatment. The high frequency of resistance observed in these antibiotics could be attributed to their use in treatment of diseases in animals and humans. The low activity of these antibiotics can also be attributed in part to earlier exposure of the isolates to these drugs, which may have enhanced resistance development. ${ }^{24}$ No one of the isolates was susceptible to all of the tested antibiotics and also none of the $S$. aureus isolates was resistant to all the tested antibiotics.

\section{Conclusion}

The present study showed that there is a high prevalence of $S$. aureus and an alarming level of resistance to commonly used antibiotics. Thus, adequate preventative and control measures are needed to reduce transmission and infections caused by resistant strains of $S$. aureus. There is need to carry out periodic monitoring of AST of important pathogens including S. aureus. It is also time to install greater control and rational use of antibiotics in order to slow down the rate of resistance development and spread of resistant organisms in the community.

\section{Acknowledgments}

We would like to thank the Catholic University of Rwanda (CUR) for its support on materials used during this study. Our gratitude is also extended to CHUK administration for the permission and assistance provided especially the Laboratory facilities and its personnel.

\section{Conflicts of interest}

The authors declare there is no conflict of interest.

\section{Funding}

None.

\section{References}

1. Chambers HF, Deleo FR. Waves of resistance:Staphylococcus aureus in the antibiotic era. Nat Rev Microbiol. 2009;7(9):629-641.

2. Monsen T, Olofsson C, Ronnmark M, et al. Clonal spread of staphylococci among patients with peritonitis associated with continuous ambulatory peritoneal dialysis. Kidney Int. 2000;57(2):613-618. 
3. Kluytmans J, Belkum A, Verbrugh H. Nasal Carriage of Staphylococcus aureus: Epidemiology, underlying mechanisms, and associated risks. Clin Microbiol Rev. 1997;10(3):505-520.

4. Perez-Vazquez M, Vindel A, Marcos C, et al. Spread of invasive Spanish Staphylococcus aureus spa-type 067 associated with a high prevalence of the aminoglycoside-modifying enzyme gene ant (4')-Ia and the efflux genes msrA/msrB. Journal of Antimicrobial Chemotherapy. 2009;63:21-31.

5. Diekema DJ, Pfaller MA, Schmitz FJ, et al. Survey of infections due to Staphylococcus species:frequency of occurrence and antimicrobial susceptibility of isolates collected in the United States, Canada, Latin America, Europe, and the Western Pacific region for the SENTRY Antimicrobial Surveillance Program, 1997-1999. Clin Infect Dis. 2001;32(Suppl 2):S114-S132.

6. Agvald-Ohman C, Lund B, Edlund CS. Multiresistant coagulasenegative Staphylococci disseminate frequently between intubated patients in a multidisciplinary intensive care unit. Crit Care. 2004;8(1):42-47.

7. Wang SH, Khan Y, Hines L, et al. Methicillin-resistant Staphylococcus aureus sequence 239-III, Ohio, USA, 2007-2009. Emerg Infect Dis. 2012;18(10):1557-1565.

8. De Angelis G, Restuccia G, Venturiello S, et al. Nosocomial acquisition of methicillin-resistant Staphyloccocus aureus (MRSA) and extendedspectrum beta-lactamase (ESBL) Enterobacteriaceae in hospitalised patients:a prospective multicenter study. BMC Infectious Diseases. 2012;12:74-79.

9. Deleo FR, Otto M, Kreiswirth BN, et al. Communityassociated methicillin-resistant Staphylococcus aureus. Lancet. 2010;375(9725):1557-1568.

10. Crisóstomo MI, Westh H, Tomasz A, et al. The evolution of methicillin resistance in Staphylococcus aureus Similarity in genetic Backgrounds in historically early methicillin-susceptible and -resistant isolates and contemporary epidemic clones. Critic Care. 2004;8:42-47.

11. Maranan MC, Moreira B, Boyle-Vavra S, et al. Antimicrobial resistance in Staphylococci. Epidemiology, molecular mechanisms and clinical relevance. Infect Dis Clin North Am. 1997;11(4):813-849.

12. Fitzroy AO. The Emergence of Mupirocin Resistance among the Clinical Isolates of Methicillin-Resistant Staphylococcus aureus in Trinidad: a First Report. Jpn J Infect Dis. 2008;61(2):107-110.

13. Lee MC, Rios AM, Aten MF, et al. Management and outcome of children with skin and soft tissue abscesses caused by communityacquired methicillin-resistant Staphylococcus aureus. Pediatr Infect Dis J. 2004;23(2):123-127.

14. Livermore DM. Antibiotic resistance in Staphylococci. Int J Antimicrob Agents. 2000;16(Suppl 1):S3-S10.

15. Adebayo OS, Edet EU, Johnson L. Phenotypic and molecular characterization of Staphylococcus aureus isolates expressing lowand high-level mupirocin resistance in Nigeria and South Africa. BMC Infect Dis. 2009;9:10-17.
16. Ekundayo EO, Ndubuisi RN. In vitro antimicrobial susceptibility pattern of Staphylococcus aureus isolates in Umuahia, Abia State, Nigeria. Afr J Clin Exper Microbiol. 2015;16(1):62-66.

17. Wilkinson BJ. Biology. In: Crossley KB, Archer GL, editors. The staphylococci in human disease. Churchill Livingstone, New York, USA, 1997. p. 1-38.

18. Collee JG, Miles RS, Watt B. Tests for identification of bacteria. In: Collee JC, Fraser AG, et al. editors. MacKie \& McCartney's Practical Medical Microbiology, 14th edn, Churchill Livingstone, New York, USA, 1996. pp. 131-149.

19. Cheesbrough M. District Laboratory Practice in Tropical Countries. 2nd edn, Part 2, Cambridge University press, USA, 2006. p. 38-65.

20. Benson HJ. Microbiological applications: Laboratory Manual in General Microbiology. Short version. 8th edn, MacGraw Hill, Boston, MA, USA, 2002. pp. 256-261.

21. Koneman EW, Allen S, Janda WM, et al. Staphyloccoci and releated organisms. Color Atlas and Textbook of Diagnostic Microbiology. 5th edn, Lippincott, Philadelphia, USA, 1997. pp. 539-576.

22. Ekundayo EO, Omodamiro OD. Evaluation of the quality of locally manufactured antimicrobial susceptibility testing discs in South Eastern Nigeria. Afr J Clin Exper Microbiol. 2008;9(3):122-128

23. Zerfie T, Moges T, Mucheye G. Staphylococcus aureus and its Antimicrobial Susceptibility Pattern in Patients, Nasalcarage of Health Personnel, and objects at Dessie referral hospital, Northern Ethiopia. Global Journal of Medical research: C Microbiology and Pathology. 2014;14(2):1-8.

24. Chijioke A, Vivian NN, Christian UO. Prevalence and Antibiotic Susceptibility Pattern of Staphylococcus aureus Isolated from Various Clinical Specimens in South East Nigeria. MOJ Cell Sci Rep. 2016;3(2):00054.

25. Grassi GG. Infections by Gram-positive bacteria: an overview. $J$ Antimicrobiol Chem. 1988;21(Suppl 1):1-7.

26. Syed ZB, Safia A Naheed Z. Antimicrobial susceptibility pattern of Staphylococcus aureus on clinical isolates and efficacy of laboratory tests to diagnose MRSA:a multi-centre study. J Ayub Med Coll Abbottabad. 2011;23(1):139-142.

27. Esan CO, Famurewa O, Lin J, et al. Characterization of Staphylococcus aureus isolates obtained from healthcare institutions in Ekiti and Ondo States, South-Western Nigeria. Afr J Microbiol Res. 2009;3(12):962968.

28. Shittu AO, Udo EE, Lin J. Phenotypic and molecular characterization of Staphylococcus aureus isolates expressing low-and-high-level mupirocin resistance in Nigeria and South Africa. BMC Infect Dis. 2009;9:10.

29. Clinical and Laboratory Standards Institute. Performance Standards for Antimicrobial Susceptibility Testing; Twenty-First Informational Supplement. M100-S21. Clinical and Laboratory Standards Institute, USA, 2011;31(1):1-188. 